\title{
Self-associating block copolymer networks for microchip electrophoresis provide enhanced DNA separation via "inchworm" chain dynamics
}

\author{
Thomas N. ChiesI ${ }^{\star}$, Karl W. Putz ${ }^{\star}$, Meena Babu*, Patrick Mathias ${ }^{\dagger}$, Kashan A. Shaikh $^{\dagger}$, \\ Edgar D. Goluch ${ }^{\dagger}$, Chang Liu ${ }^{\dagger}$, and Annelise E. Barron ${ }^{*}$ \\ "Department of Chemical and Biological Engineering, Northwestern University, Evanston, Illinois \\ 60208 \\ tDepartment of Electrical and Computer Engineering, Micro and Nanotechnology Laboratory, \\ University of Illinois at Urbana-Champaign, Urbana, IL 61801
}

\begin{abstract}
We describe a novel class of DNA separation media for microchip electrophoresis, "physically crosslinked" block copolymer networks, which provide rapid $(<4.5 \mathrm{~min})$ and remarkably enhanced resolution of DNA in a size range critical for genotyping. Linear poly(acrylamide-codihexylacrylamide) (LPA-co-DHA) comprising as little as $0.13 \mathrm{~mol} \%$ dihexylacrylamide yields substantially improved electrophoretic DNA separations compared to matched molar mass linear polyacrylamide. Single-molecule videomicroscopic images of DNA electrophoresis reveal novel chain dynamics in LPA-co-DHA matrices, resembling inch-worm movement, to which we attribute the increased DNA resolution. Substantial improvements in DNA peak separation are obtained, in particular, in LPA-co-DHA solutions at polymer/copolymer concentrations near the interchain entanglement threshold. Higher polymer concentrations yield enhanced separations only for small DNA molecules ( $<120$ base-pairs). Hydrophobically crosslinked networks offer advantages over conventional linear polymers based on enhanced separation performance (or speed) and over chemically crosslinked gels because hydrophobic crosslinks can be reversibly broken, allowing facile microchannel loading.
\end{abstract}

\section{Introduction and Background}

The sequencing of the human genome was in part accomplished through engineering achievements in polymer science, and followed a paradigm shift in electrophoresis technology from slab gels ${ }^{1}$ to capillary electrophoresis. This shift is continuing with a movement towards microfluidic chips ${ }^{2,3}$ and for these miniaturized platforms, there is a need for high-performance biomolecule separation media that can be loaded into microdevices with ever-decreasing footprints. For high-performance genetic analysis on chips, a fresh "gel" is required for each analysis. Covalently crosslinked polyacrylamide gels, once the gold standard for electrophoretic DNA separations, are monolithic and cannot be loaded and reloaded into microchannels, while in vitro polymerization of acrylamide in confined microchannels yields poor separation media. To address this problem, various "replaceable" linear polymer and copolymer solutions have been developed as DNA separation media for capillary and microchip electrophoresis systems. Among notable

Corresponding Author: Annelise E. Barron, Associate Professor, Northwestern University, Department of Chemical and Biological Engineering and (by courtesy) Chemistry, 2145 Sheridan Road, Room E136, Evanston, Illinois 60208. Tel: (847) 491-2778, Fax: (847) 491-3728, a-barron@ northwestern.edu. 
approaches taken to improve microchannel DNA separations using pre-formed polymers are the use of entangled solutions of high-molar mass linear polymers (particularly polyacrylamide ${ }^{4-6}$ and polydimethylacrylamide ${ }^{7,8}$ ), as well as novel polymers with diverse non-linear architectures and properties (e.g. thermo-responsive linear copolymer networks, ${ }^{9,} 10$ branched copolymers, ${ }^{11}$ and sparsely crosslinked nanogel solutions ${ }^{12}$ ). Here we present a new class of DNA separation media based on hydrophobically crosslinked networks of block copolymers of $N, N$-dialkylacrylamides with acrylamide, which we have tailored for implementation in microchip-based DNA analysis systems and which provide enhanced DNA separations as a result of the distinct DNA migration dynamics they engender. In previous work with these copolymers, aimed at purifying DNA away from albumins, ${ }^{13}$ we noticed that LPA-co-DHAs can provide a dramatic increase in DNA resolution. Here, we explore the mechanisms responsible for this increased resolution via high-field microchannel electrophoresis and single-molecule epifluorescent videomicroscopy.

\section{Materials and Methods}

The various monomers, polymers and copolymers reported here were synthesized, purified and characterized as described in our recent publication in which they were applied for another purpose. ${ }^{13}$ For matrices studied here, polymer and copolymer molar mass were controlled via the addition of a chain transfer agent to the polymerization (isopropanol). While LPAs were made by standard aqueous-phase polymerization of acrylamide in the absence of crosslinker, the LPA-co-DHAs were synthesized by micellar polymerization using SDS. The resultant polymer and copolymer molar masses were analyzed by tandem GPC-MALLS as previously described ${ }^{13}$ with a modified mobile phase (33 vol\% methanol in water) for the block copolymers.

Capillary electrophoresis experiments were conducted with UV detection using a BioRad Biofocus 3000 (BioRad, Hercules, CA). Capillaries were $25 \mathrm{~cm}$ in length total, with $20-\mathrm{cm}$ effective length and a 75- $\mu \mathrm{m}$ inner diameter. Capillaries were pretreated, on the instrument, with a 2-minute $1 \mathrm{M} \mathrm{HCl}$ high-pressure (100 psi) pre-rinse followed by a 2-minute highpressure rinse of a dilute $(0.25 \mathrm{wt} \%)$ poly- $N$-(hydroxyethylacrylamide) coating solution. ${ }^{14}$ The capillary was then allowed to rest for 15 minutes to facilitate coating formation, after which the desired separation matrix was loaded into the capillary using 120 psi applied pressure. The applied electric field was $300 \mathrm{~V} / \mathrm{cm}$ with a typical current of $17 \mu \mathrm{A}$ during electrophoretic separations. Double-stranded DNA samples were purchased from Invitrogen (San Diego, CA) and consisted of 10, 50, and 100 base pair ladders. DNA was diluted to 10 $\mu \mathrm{g} / \mathrm{ml}$ in reverse-osmosis purified, deionized water and injected with an applied electric field of $300 \mathrm{~V} / \mathrm{cm}$ for 7 seconds. For microchip electrophoresis experiments the DNA was stained with $1 \mu \mathrm{M}$ ethidium bromide. Microchip electrophoresis was carried out using a custombuilt laser-induced fluorescence detector described previously. ${ }^{13}$ T8050 glass microchips (Micronit, Enschede, The Netherlands) with an 8-cm detection length were adsorptively coated with a dilute poly- $N$-(hydroxyethylacrylamide) solution for 5 minutes following a 5minute $1 \mathrm{M} \mathrm{HCl}$ pretreatment. DNA injection into the offset $\mathrm{T}$ of the microchip was accomplished by grounding the sample reservoir and applying a $500 \mathrm{~V} / \mathrm{cm}$ field to the sample waste reservoir for 30 seconds. The separation field strength was $300 \mathrm{~V} / \mathrm{cm}$ and pullback conditions were applied to both sample and sample waste to avoid DNA sample leakage into the analysis channel.

Single molecules of DNA were visualized using a sensitive high-speed imaging system created especially for observation of DNA electrophoresis under high electric fields, similar to a previously reported system. ${ }^{15,16}$ Our setup consists of a 100-watt mercury lamp light source directed into a Nikon TE200 inverted epifluorescence microscope. The light is 
focused and passed through a heat-absorbing filter. The light is filtered via a standard FITC filter cube (Chroma Technology, Brattleboro, VT), reflected off a dichroic mirror and focused through a Nikon CFI 100× N.A. 1.4, $0.133 \mathrm{~mm}$ working distance apochromatic oilimmersion microscope objective. The collected light is then directed into a VS4-1845 Generation 3 image intensifier (Videoscope International, Dulles, VA) where it is focused onto a 0.5 inch CCD, TM-6710-CL camera (JAI Pulnix, Sunnyvale, CA). This high-speed camera has an adjustable frame rate of up to 120 frames per second at the full resolution of $648 \times 484$ pixels. Videos and images are directly captured onto a computer (Dell, $3 \mathrm{GHz}$ processor, 1 Gigabyte of memory) via camera link technology to a PIXCI control board (EPIX INC., Buffalo Grove, IL) and the capture is controlled through XCAP-STD (EPIX INC, Buffalo Grove, IL) software. Videos were adjusted for brightness and contrast using Adobe Premier 6.5 ${ }^{\mathrm{TM}}$ and individual frames were exported to Adobe Photoshop 7.0 ${ }^{\mathrm{TM}}$ for background subtraction and image smoothing.

For single-molecule videomicroscopy studies, linearized $\lambda$-phage DNA (48.5 kbp, N3011S, New England Biolabs, Ipswich, MA) was fluorescently stained ${ }^{17}$ with YOYO-1 (Molecular Probes / Invitrogen, San Diego CA) replacing TOTO. Catalase, Glucose Oxidase (Fisher Scientific, Pittsburgh, PA) and $\beta$-mercaptoethanol (Sigma-Aldrich, St. Louis, MO) are added into the solution to reduce the amount of detrimental oxygen and free-radical interactions with DNA when exposed to air and light. ${ }^{18}$ Pipette tips were cut wider with a razor blade to avoid shearing and breaking the DNA. Microchannels used for DNA imaging are composed of a Sylgard 184 poly(dimethylsiloxane) (Fisher Scientific, Pittsburgh, PA), or PDMS, top piece and a glass cover slip of thickness number 1.5 (Fisher Scientific, Pittsburgh, PA). The PDMS top piece is formed by first mixing PDMS pre-polymer and curing agent at a 10:1 ratio (by weight) respectively, degassing the mixture and then pouring this mixture on top of thinly cut (1-2 mm) slices of Scotch Magic Tape ${ }^{\mathrm{TM}}$ (50-60 $\mu \mathrm{m}$ thickness) or a photolithographically defined SU-8 mold with size ( $4 \mathrm{~cm}$ by $50 \mu \mathrm{m}$ by $50 \mu \mathrm{m})$. After allowing the PDMS to cure in a vacuum chamber overnight it is then cut to fit on a cover slip, peeled off and annealed to the glass cover slips. The stained DNA-polymer solution is then pipetted onto one end of the microchip and allowed to wick into the microchannel by capillary action. Another drop of solution was placed at the other end to serve as a buffer reservoir. The electric field is applied via platinum-tipped electrodes powered by a number of 9-volt batteries connected in series to give the desired electric field.

\section{Results and Discussion}

Hydrophobically crosslinked networks can be formed by the physical association of $N$-alkyl moieties present in small blocks in overall water-soluble, acrylamide-based copolymers with predominantly hydrophilic backbones and small $(<5 \mathrm{~mol} \%)$ amounts of the hydrophobically modified co-monomers. ${ }^{19,20}$ The fundamental rheological behavior of this class of block copolymers has been studied in the past, with the suggestion that they could be useful for oil recovery by polymer flooding of spent oil fields. Here, we present copolymers comprised of acrylamide and $N, N$-dihexylacrylamide (LPA-co-DHAs), comprising very tiny amounts of the hydrophobic co-monomers, as a new class of physically crosslinked matrices for microchannel electrophoretic separations of DNA. Monomer structures are illustrated in Figure 1A.

In LPA-co-DHAs synthesized by micellar polymerization, the hydrophilic acrylamide monomers form the bulk of the polymer backbone while small blocks of hydrophobic dihexylacrylamide monomers can form either intramolecular or intermolecular physical entanglements, dependent on the concentration and the molar mass of the copolymer. ${ }^{21,22}$ These different copolymer conformation/association regimes are marked by two critical concentration values: $\mathrm{C}_{\eta}$, a concentration similar in meaning to $\mathrm{c}^{*}$ (the polymer coil overlap 
threshold) in unmodified polymers, and $\mathrm{C}_{\mathrm{T}}$, the concentration at which "elastic" behavior becomes dominant for unmodified polymer chains in solution. ${ }^{23,} 24$ Typical intra and interchain conformations and interactions in these different regimes are highly relevant to their use as DNA separation media, and are illustrated in Figure 1B. Previous studies have shown that $\mathrm{C}_{\eta}$ exhibits a strong dependence on the molecular weight of the copolymers, but is independent of the block length and the mole percentage ( $\mathrm{mol} \%)$ of the hydrophobic moiety. The concentration regime between $C_{\eta}$ and $C_{T}$ is typically marked by a sharp linear increase in solution viscosity, depending on the mol \% hydrophobe and the average dihexylacrylamide block length. At concentrations below $\mathrm{C}_{\eta}$, LPA-co-DHA polymers are in a relatively dilute, unentangled state, and dihexylacrylamide units tend to form intramolecular bonds within the polymer chain. Polymer chain contraction occurs because of these intramolecular associations, resulting in a slightly lower solution viscosity relative to an unmodified LPA solution with matched LPA molar mass and concentration. As copolymer concentration is increased beyond $\mathrm{C}_{\eta}$, individual polymer molecules begin to interact and entangle with each other, and the dihexylacrylamide blocks within the copolymers associate intermolecularly via hydrophobic bonding. ${ }^{24}$ Alternatively, homopolymer networks without hydrophobic blocks will remain mostly disassociated and have a loose network structure in this concentration range. As polymer or copolymer concentration is increased to values near $\mathrm{C}_{\mathrm{T}}$, individual polymer or copolymer coils begin to truly overlap in solution and form an infinite entangled network. The LPA-co-DHA copolymer system forms a more robust network near this concentration due to the hydrophobic associations of the dihexylacrylamides that physically crosslink the network. As we will discuss, the concentration $\mathrm{C}_{\mathrm{T}}$ appears to be most critical for enhanced DNA separations in physically crosslinked polymer solutions.

As polymer or copolymer concentration is further increased, the scaling of the viscosity is dominated by the effects of increased polymer concentration. At these higher concentrations LPA and LPA-co-DHA polymer solution viscosities scale similarly and have reptationdominated dynamics. A plot of zero-shear viscosity vs. polymer concentration can be seen in Figure 2, where the viscosities of matched molar mass (1.3 million $\mathrm{g} / \mathrm{mol}$ ) LPA solutions are compared to LPA-co-DHA comprising $0.23 \mathrm{~mol} \%$ dihexylacrylamide. Because the copolymers presented in this work have minimal amounts of dihexylacrylamide (compared to those reported by Candau et. $a l^{21,22}$ ) the viscosity differences in the region between $\mathrm{C}_{\eta}$ and $\mathrm{C}_{\mathrm{T}}$, while appreciable, are not as well pronounced. $\mathrm{C}_{\eta}$ and $\mathrm{C}_{\mathrm{T}}$ were determined to be approximately $0.15 \mathrm{wt} \%$ and $2.5 \mathrm{wt} \%$, respectively. In addition to $\mathrm{C}_{\mathrm{T}}$, a concentration threshold we have designated in Figure 2 as $\mathrm{C}_{\mathrm{ES}}$ (ES for equal separation) is delineated at 4.6 wt \% copolymer; and is correlated to similar polymer/copolymer network strengths (discussed below).

With regard to rheological behavior, a major advantage to physically crosslinked polymer solutions, such as LPA-co-DHAs, is that physical crosslinks can be disassociated under applied shear, such as during pressurized microchannel loading. Moreover, the entangled copolymer network shear-thins in solution as chains become forcefully disentangled from each other and orient in the flow field, so that the solution viscosity decreases by several orders of magnitude under moderate applied shear (such as those typically used for the pressurized loading of microchannels). This behavior is unlike that of covalently crosslinked networks, which are monolithic and do not shear-thin and are extremely difficult, if not impossible, to load into a microchannel. Upon cessation of shear, LPA-co-DHA copolymer chains relax and the dihexylacrylamide units will re-associate, thus returning the network to a physically crosslinked state.

Electrophoresis of DNA in matrices with polymer concentrations in each of these regimes yields interesting DNA separation performance results, which can be correlated with the 
different polymer network architectures. We compared the results of microchannel electrophoresis separations of mixtures of DNA molecules with different chain lengths ("DNA ladders") performed in LPA and LPA-co-DHA solutions at $3 \mathrm{wt} \%$, a concentration near $\mathrm{C}_{\mathrm{T}}$, to study the ramifications of hydrophobic crosslinking. At this concentration, the block copolymer network is both highly (though reversibly) crosslinked and physically entangled. An LPA-co-DHA copolymer with a weight-average molar mass of 1.3 million g/ mol has approximately 18,000 repeat units. The copolymer with the smallest dihexylacrylamide content we studied $(0.13 \mathrm{~mol} \%)$, has on average 20 dihexylacrylamide units spread among 4 or 5 blocks, which are available to physically crosslink a given polymer chain with neighboring copolymer chains.

Figure 3 presents several capillary electropherograms of DNA separations obtained under identical conditions through LPA-co-DHA copolymer solutions and a matched-molar mass LPA homopolymer dissolved at $3 \mathrm{wt} \%$. In both panels of the figure, the data have been normalized and offset for clarity, and in Figure 3B the peaks are time-shifted to clarify the effect of copolymer content on DNA resolution (see figure caption). Polymer concentration and the extent of interchain polymer entanglements are very important: we found that the separation of DNA molecules in the 50 base-pair ladder in $3.0 \mathrm{wt} \%$ LPA is substantially improved relative to what was seen for lower concentrations (e.g. $1.5 \mathrm{wt} \% \mathrm{LPA})$, with better peak spacing and sharper peak shapes, to about 650 bases, with the first peak eluting at 4 min (data not shown). Separations performed in LPA-co-DHA networks yield slower separations than in an LPA homopolymer network at the same concentration. As dihexylacrylamide monomer content increases, the migration time of the DNA becomes slightly longer, as seen in Figure 3A. However, the ability of the LPA-co-DHA network to separate DNA of differing sizes is significantly better than in the homopolymer network, and is nearly independent of dihexylacrylamide content as seen in Figure 3B. In the LPA-coDHAs, DNA peak spacing is particularly improved for DNA smaller than 450 bases, and is moderately improved for DNA with sizes greater than 450 bases. Peak efficiencies for both (co)polymer systems are comparable at 300,000 plates / meter; however, the peak selectivity for the copolymer systems is improved by $~ 15 \%$ on average in this DNA size range. Interestingly, the presence of the hydrophobic dihexylacrylamide monomer at concentrations up to $0.43 \mathrm{~mol} \%$ does not seem to adversely affect DNA peak efficiencies; however, the best overall separation is obtained in the LPA-co-DHAs with the smallest amount of dihexylacrylamide, $0.13 \mathrm{~mol} \%$ (red trace). The spiked peak in Figures 3A and $3 \mathrm{~B}$ representing " 350 " base pairs is split into two separate peaks that are fairly well resolved. This "extra" peak is not present in the LPA matrix and only appears as a shoulder in the other LPA-co-DHA matrixes. The source of this peak is likely an imprecise product of the enzymatic cleavage used to produce this ladder. By plotting migration time vs. DNA size we estimated the size of the spiked peak and the "extra" peak to be 350 and 360 base pairs. The increased resolution in LPA-co-DHA can also be seen by comparing elution time differences between the 50-base pair fragment and the spiked 350-base pair fragment in Figure 3B. In the LPA-co-DHA matrices there is an additional half-minute in elution time and the 350-base pair fragment elutes at the same relative time as the 550-base pair fragment in LPA. For these shorter DNA fragments, the increase in the robustness of the polymer network is expected to provide enhanced separation via Ogston like sieving. This is an increase in resolution of $40 \%$, i.e., a very significant improvement in the ability to determine accurate DNA sizes in this critical size range for genotyping, which has never before been obtained in linear polymer systems where the acrylamide backbone has been substituted with another monomer.

Interestingly, at concentrations above the hydrophobic entanglement region, near $\mathrm{C}_{\mathrm{ES}}$ in Figure 2, the enhanced separation effect is diminished. At $\mathrm{C}_{\mathrm{T}}$, the LPA matrix begins to become a truly entangled polymer solution and the extent of network connectivity is similar 
to that of the LPA-co-DHAs at the same concentration. The separation performance of the two systems (LPA and LPA-co-DHA) should thus become comparable again. At concentrations near $\mathrm{C}_{\mathrm{ES}}$ the separations are equivalent for approximately the first 400 base pairs (tested with a 50-bp and 100-bp ladder, data not shown) while the separation quality diminishes for DNA sizes greater than 400 bases due to a more constricted polymer network and the onset of biased reptation. However, the separation performance is substantially better in the LPA-co-DHAs for DNA with molecular sizes less than 120 base pairs, as shown in Figure 4. Specifically, Figure 4 presents two microchip electropherograms showing the separation of a 10-base pair ladder (10 to 330 bases with the 100-bp fragment spiked) separated in $5 \mathrm{wt} \%$ LPA or LPA-co-DHAs, both with molar masses 1.3 million g/ mol. Both polymer matrices separate the 10-bp DNA ladder well (providing 1.4 million and 2.8 million plates/meter for DNA peaks in LPA and LPA-co-DHA respectively), and with nearly equal peak spacing. However, the electropherogram for the LPA-co-DHA matrix has extra peaks, seen as doublets, which are not clearly resolved in the LPA homopolymer matrix. Again these extra peaks reveal side products of the enzymatic cleavage procedure and represent a few base-pairs difference in DNA size, demonstrating the additional separation power of the LPA-co-DHA networks for smaller sized DNA, which may be important in applications such as forensic short-tandem repeat (STR) sizing. As many as five or six peaks can be seen for the 90- and 100-base pair fragments in the LPA-co-DHAbased separation, whereas only the one broad peak is seen in the LPA electropherogram. In this concentration range the LPA-co-DHA matrix comprises a more tightly associated network with a smaller average mesh size than the LPA matrix, and hence separates smaller fragments of DNA better. The data shown in Figure 4 also demonstrate our ability to load these matrices, which have high zero-shear viscosities but which also show shear-thinning behavior, into microfluidic devices with small channels. These separations performed in microchips are extremely fast (260 seconds) and are obtained with excellent resolution.

Electrophoresis of DNA was done in copolymer solutions with concentrations below the critical value for interchain entanglements, $C_{T}$ (Supplementary Figure 1). In both the homopolymer and the copolymer networks, DNA molecules are well separated up to $\sim 600$ base pairs and no significant differences in peak resolution between separations in LPA vs. LPA-co-DHA networks are seen in this concentration range. However, the migration time of the DNA is monotonically decreased with increasing copolymer hydrophobicity. We attribute the increase in speed for more hydrophobic matrices to the copolymer chain contraction associated with intramolecular bonding. A copolymer chain in contraction occupies less physical volume and will have a lower frequency of collisions with electromigrating DNA molecules, and hence allow faster DNA migration. A copolymer chain in this state may also be slightly more "effective" at entangling with a DNA molecule during a collision, so that the combination of the two effects gives approximately the same resolution. Additionally, as discussed below, a novel separation mechanism has been discovered when DNA is separated through LPA-co-DHAs networks that are predominantly in the physically crosslinked state, yielding slightly faster separations.

We used single-molecule epifluorescent videomicroscopy to observe the motion of DNA molecules through the various polymer solutions. Evidence suggesting a unique modality of separation, absent in conventional polymer solutions, is presented in Figure 5. Image captures from these videos of a single molecule of fluorescently labeled DNA migrating via the two well-known mechanisms of DNA separation, transient entanglement coupling 25,26 (left) and reptation ${ }^{9,27}$ (top) are shown for two representative polymer solutions (a 0.3 wt \% solution of 1.3 million $\mathrm{g} / \mathrm{mol}$ LPA, and a $1.8 \mathrm{wt} \%$ solution of $1.3 \mathrm{million} \mathrm{g} / \mathrm{mol}$ LPA). The field strengths were approximately $130 \mathrm{~V} / \mathrm{cm}$ in each case. In the first mechanism of separation, transient entanglement coupling, a DNA molecule becomes entangled with dissolved polymer, stretches out to form a characteristic "U" shape oriented in the direction 
of the electric field, and physically drags the polymer with it until the DNA molecule or polymer chain slides off and discontinues the entanglement. After the collision event the DNA chain contracts back into a globular conformation. Dots were superimposed over the video frame-captures to demonstrate how the entangled DNA-polymer conjugate travels in the direction of the electric field with time. In the second mechanism of DNA separation, reptation, DNA molecules snake through a highly entangled polymer matrix continuously, in an elongated conformation. Because the average mesh size of the polymer network is much smaller than the DNA coil size, DNA molecules migrate through the network "head-first," and must meander their way through the tortuous path of the entangled polymer matrix to travel in the direction of the electric field. Imaging experiments in a chemically crosslinked poly(acrylamide) gel $^{28}$ were conducted as well, and also showed reptation-like DNA chain dynamics. Although reptation has been much discussed in the literature, ${ }^{29,30}$ to our knowledge, Figure 5 presents the first published images of electrophoretically driven DNA reptation through a linear polymer solution

When we imaged DNA migration through LPA-co-DHA solutions, we discovered that DNA can also migrate through a polymer matrix by a previously undiscovered mechanism which we call "stationary entanglement coupling". The stationary entanglement coupling migration mode can be seen in Figure 5 (bottom-right panel) and resembles a DNA collision event with a photolithographically defined insulating post. ${ }^{31}$ Dots are superimposed to show the stationary nature of the chain entanglement point (which must be the hydrophobically associated DHA domains). The LPA-co-DHA used for the electrophoresis experiment shown in Figure 5 shown has an average molar mass of 4.3 million $\mathrm{g} / \mathrm{mol}$ and comprises just $0.17 \mathrm{~mol} \%$ dihexylacrylamide. We used this copolymer at a $0.75 \mathrm{wt} \%$ polymer concentration, which is within the region of hydrophobic association for this higher molar mass sample. In stationary entanglement coupling, a DNA molecule moves in the direction of the electric field in a globular conformation until it collides with a polymer chain. Both the DNA and (apparently) the polymer chain on which DNA is entangled remain stationary at the collision point, while the two ends of the DNA chain extend and stretch around the polymer to form a characteristic " $\mathrm{J}$ " shape. Both sides of the "J"-shaped DNA chain compete in this stretching event and extend away from the collision/entanglement point. The longer side of the "J" eventually wins out and pulls the small end of the DNA around the point of collision. After the DNA molecule disassociates from the polymer obstacle it snaps back into a globular shape. Examining the DNA with real-time videomicroscopy images shows a mode of DNA locomotion very similar to an inchworm or a Slinky ${ }^{\mathrm{TM}}$ with many repetitive and dramatic chain stretching and contraction events (video available with supplementary information).

The LPA-co-DHA copolymer network used in Figure 5 is relatively open with ample spacing between chains as evidenced by these chain dynamics, and the average relaxation time of the polymers (determined by analysis of storage and loss moduli data, not shown) is $\sim 7.9$ seconds. For an electric field strength of $130 \mathrm{~V} / \mathrm{cm}$, the collision time is on the order of 0.5 seconds and hence the LPA-co-DHA network behaves like a rigid crosslinked structure, which does not allow the DNA to drag the polymer along with it during electrophoresis. The videomicroscopy results therefore provide complimentary evidence to the rheological data to validate the existence of a physically crosslinked polymer architecture. This novel separation mechanism should be seen for all polymers in this class of physically crosslinked polymer networks when within a region of hydrophobic association between $C_{\eta}$ and $C_{T}$. As the concentration of the copolymer is increased, the average mesh size of the network decreases and the frequency of DNA-polymer collisions increases. At sufficiently high polymer concentrations the DNA begins to experience multiple collision events while stretched, and must wrap around multiple polymer chains to continue moving in the direction of the electric field. Thus, the DNA molecules begin to snake through the matrix 
approaching traditional reptation migration dynamics. Further investigations of the transition from one migration mechanism to another are ongoing, and representative real-time videos of each mechanism can be found in the supplementary information.

\section{Conclusions}

Physically crosslinked polymer solutions, despite their markedly increased viscosity, can be loaded into capillaries and microfluidic devices under moderate applied pressure and yield rapid, high-resolution DNA separations. These matrices can be tailored to yield faster DNA separations with resolution equal to that provided by conventional LPA matrices, or dramatically enhanced DNA resolution with the inclusion of as little as $0.13 \mathrm{~mol} \%$ dihexylacrylamide into a block copolymer. Solutions of LPA are currently considered the gold standard for DNA separations by microchannel electrophoresis, and hence it is highly significant that this new class of separation media can be tailor-made to outperform its LPA counterparts. The use of single-molecule epifluorescent videomicroscopy can help researchers to elucidate the relationship between DNA separation mechanisms and separation performance. These novel block copolymer separation media can be applied to clinical diagnostics and DNA identity assays, providing high-resolution separations for more precise genetic information, or be applied to provide faster DNA separations on chips in shorter distances thereby effectively reducing the required analysis time.

\section{Supplementary Material}

Refer to Web version on PubMed Central for supplementary material.

\section{Acknowledgments}

The authors would like to thank Patrick Doyle and Anthony Balducci of MIT for the technical assistance in the DNA imaging experiments. Financial support was provided by the Air Force Office of Scientific Research (AFOSR), the Defense Advanced Research Projects Agency (DARPA DURINT Grant No. F46920-01-1-0401), the NSF through the Northwestern University Nanoscale Science and Engineering Center (Award No. EEC-0118025), the NIH (Grants 5 R01 HG-01970-02S1 and AI-03-017).

\section{Abbreviations}

LPA

LPA-co-DHA

linear polyacrylamide

GPC-MALLS

linear poly(acrylamide-co-dihexylacrylamide)

CE gel permeation chromatography - multi angle laser light scattering capillary electrophoresis

\section{References}

(1). Sanger F, Air G, Barrell B, Brown N, Coulson A, Fiddes J, Hutchison C, Slocombe P, Smith M. Nature. 1977; 265:687-695. [PubMed: 870828]

(2). Aborn J, El-Difrawy S, Novotny M, Gismondi E, Lam R, Matsudaira P, Mckenna B, O’Neil T, Streechon P, Ehrlich D. Lab On A Chip. 2005; 5:669-674. [PubMed: 15915260]

(3). Paegel B, Emrich C, Weyemayer G, Scherer J, Mathies R. Proceedings of the National Academy of Sciences of the United States of America. 2002; 99:574-579. [PubMed: 11792836]

(4). Salas-Solano O, Carriho E, Kotler L, Milelr A, Goetzinger W, Sosic Z, Karger B. Analytical Chemistry. 1998; 70:3996-4003. [PubMed: 9784744]

(5). Detwiler M, Hamp T, Kazim A. Biotechniques. 2004; 36:932-933. [PubMed: 15211740]

(6). Zhou H, Miller A, Sosic Z, Buchholz B, Kotler L, Karger B. Analytical Chemistry. 2000; 72:1045-1052. [PubMed: 10739210] 
(7). Buchholz B, Doherty E, Albarghouthi M, Bogdan F, Zahn J, Barron A. Analytical Chemistry. 2001; 73:157-164. [PubMed: 11199960]

(8). Wang Y, Liang D, Ying O, Chu B. Electrophoresis. 2005; 26:126-136. [PubMed: 15624193]

(9). Sartori A, Barbier V, Viovy J. Electrophoresis. 2003; 24:421-440. [PubMed: 12569534]

(10). Buchholz B, Shi W, Barron A. Electrophoresis. 2002; 23(10):1398-1409. [PubMed: 12116149]

(11). Barbier V, Buchholz B, Barron A, Viovy J. Electrophoresis. 2002; 23(10):1441-1449. [PubMed: 12116154]

(12). Doherty E, Kan C, Paegel B, Yeung S, Cao S, Mathies R, Barron A. Analytical Chemistry. 2004; 76:5249-5256. [PubMed: 15362880]

(13). Chiesl T, Shi W, Barron A. Analytical Chemistry. 2005; 77:772-779. [PubMed: 15679343]

(14). Albarghouthi MN, Buchholz BA, Huiberts PJ, Stein TM, Barron AE. Electrophoresis. 2002; 23:1429-1440. [PubMed: 12116153]

(15). Carmejane, O. d.; Yamaguchi, Y.; Todorov, T.; Morris, M. Electrophoresis. 2001; 22:2433-2441. [PubMed: 11519947]

(16). Yamaguchi Y, Todorov TI, Morris MD, Larson RG. Electrophoresis. 2004; 25:999-1006. [PubMed: 15095440]

(17). Randal GC, Doyle P. Macromolecules. 2005; 38:2410-2418.

(18). Yanagida, M.; Morikawa, K.; Hiraoka, Y.; Matsumoto, S.; Uemura, T.; Okada, S. Applications of Fluorescence in the Biomedical Sciences. Alan R. Liss, Inc.; New York: 1986.

(19). Hill A, Candau F, Selb J. Macromolecules. 1993; 26:4521-4532.

(20). Biggs S, Hill A, Selb J, Candau F. Journal of Physical Chemistry. 1992; 96:1505-1511.

(21). Candau F, Regaldo EJ, Selb J. Macromolecules. 1998; 31:5550-5552.

(22). Volpert E, Selb J, Candau F. Macromolecules. 1996; 29:1452-1463.

(23). Regaldo E, Selb J, Candau F. Macromolecules. 1999; 32:8580-8588.

(24). Volpert E, Selb J, Candau F. Polymer. 1998; 39:1025-1033.

(25). Sunada W, Blanch H. Biotechnology Progress. 1998; 14:766-772. [PubMed: 9758667]

(26). Barron A, Blanch H, Soane D. Electrophoresis. 1994; 15:597-615. [PubMed: 7925237]

(27). Kantor R, Guo X, Huff E, Schwartz D. Biochemical and Biophysical Research Communications. 1999; 258:102-108. [PubMed: 10222242]

(28). Guttman, A. Beckman Coulter, Inc (Fullerton, CA); USA: 2002.

(29). DeGennes P. Journal of Chemical Physics. 1971; 55:572.

(30). Heller C. Electrophoresis. 1999; 20:1962-1977. [PubMed: 10451104]

(31). Randal GC, Doyle PS. Physical Review Letters. 2004; 93:058102. [PubMed: 15323733] 


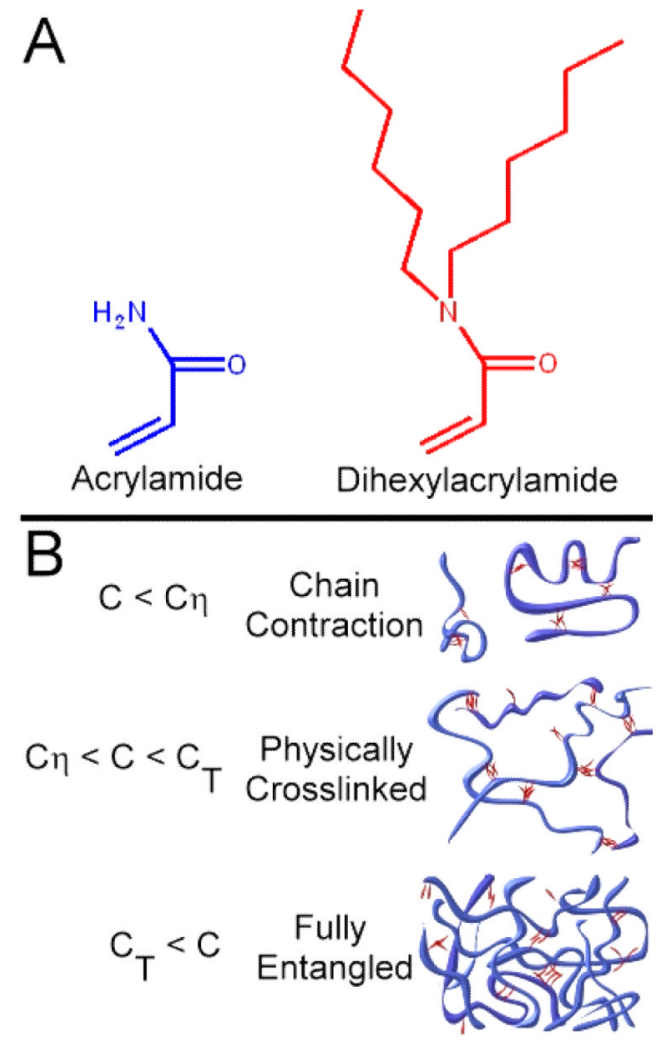

Figure 1.

Schematic illustration of monomer structures, and copolymer conformations in different concentration regimes. (A) Hydrophilic acrylamide is used to create the majority of the polymer backbone. Dihexylacrylamide becomes incorporated via randomly distributed blocks within the polymer. (B) Different copolymer conformation states for physically crosslinked copolymers. At low polymer concentrations, dihexylacrylamide forms intramolecular associations and LPA-co-DHAs exist in a state of chain contraction. At moderate concentrations, dihexylacrylamide forms intermolecular bonds and leads to the formation of a physically crosslinked network. At higher concentrations (greater than $\mathrm{C}_{\mathrm{e}}$, the entanglement concentration for unmodified polymers) both LPA and LPA-co-DHAs exist as fully entangled networks, which only the LPA-co-DHAs are physically crosslinked. 


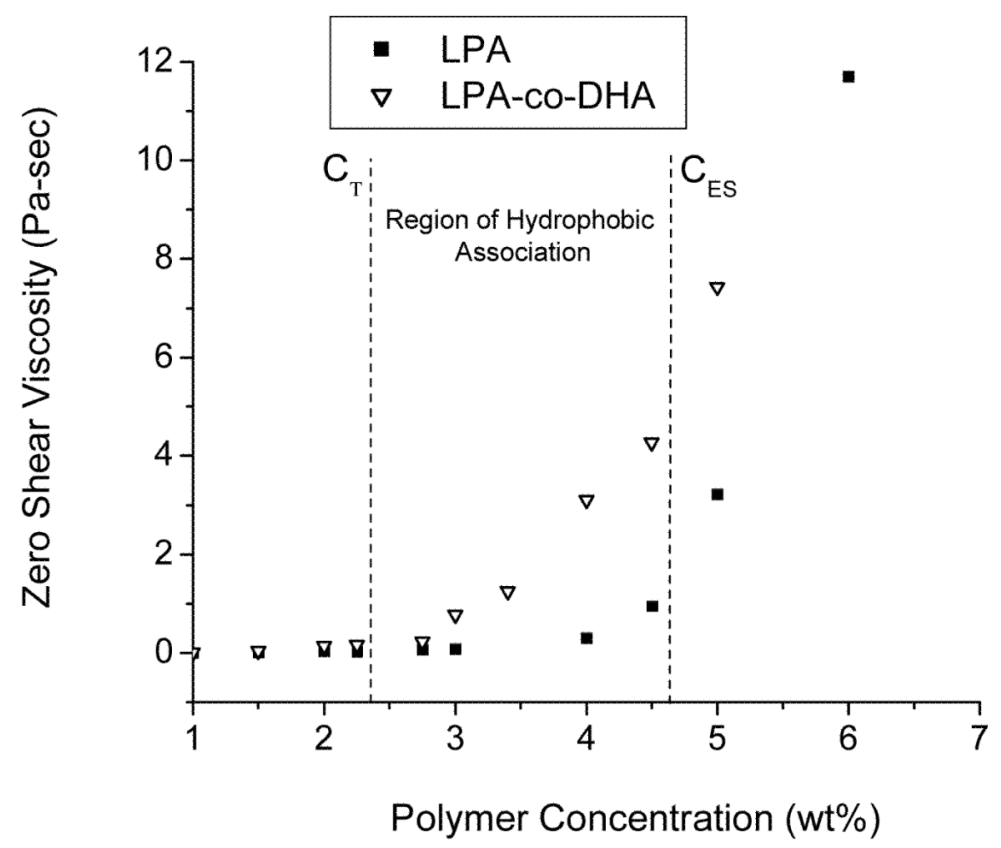

Figure 2.

Comparison of the zero-shear solution viscosities of LPA and LPA-co-dihexylacrylamide networks. The sharp increase in viscosity between $2 \mathrm{wt} \%$ and $4.5 \mathrm{wt} \%$ for the LPA-coDHA indicates the region of interchain hydrophobic chain association and also of improved DNA separation performance. Both the copolymer and LPA have weight-average molar masses of 1.3 million $\mathrm{g} / \mathrm{mol}$. The LPA-co-DHA polymers contain on average $0.23 \mathrm{~mol} \%$ dihexylacrylamide. 


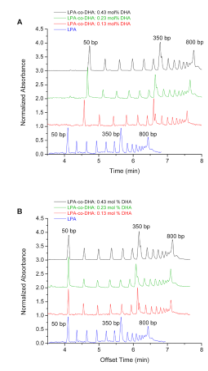

Figure 3.

Separation of a 50-bp DNA ladder in polymer solutions in the concentration range of increased separation performance. The polymers shown are all $\sim 1.3 \mathrm{million} \mathrm{g} / \mathrm{mol}$ and were used at $3 \mathrm{wt} \%$ concentrations. Data for DNA migration in the unmodified polymer network, based on LPA, are shown in blue. LPA-co-DHAs have dihexylacrylamide content of 0.13 $\mathrm{mol} \%$ (red), $0.23 \mathrm{~mol} \%$ (green), and $0.43 \mathrm{~mol} \%$ (black). Normalized raw data are shown in Figure 3A, while in Figure 3B the peaks in the LPA-co-DHAs have been time shifted such that the first DNA peaks ( $50 \mathrm{bp}$ ) are aligned with the first peak in the LPA electropherogram, to more easily show the differences in peak selectivity and resolution. 


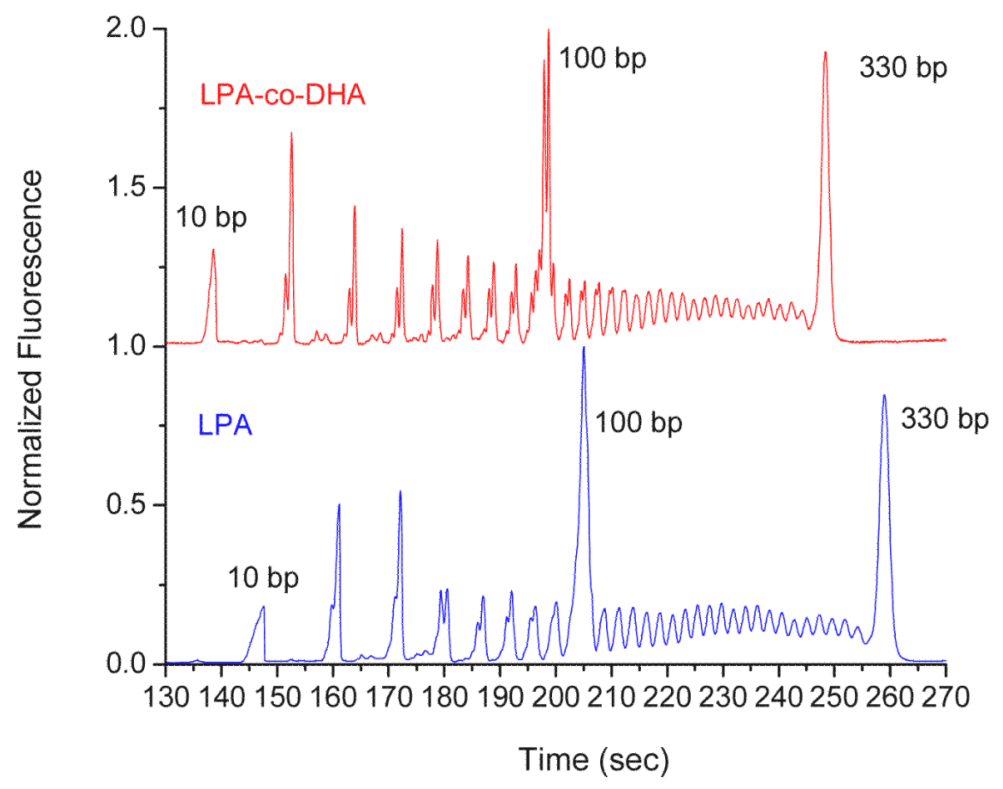

Figure 4.

Microchip electropherogram showing increased separation performance for the analysis of a 10-bp DNA ladder (as indicated by the doublets) of small DNA in a LPA-co-DHA (red) for small fragments of DNA compared to that of unmodified polyacrylamide (blue), at polymer concentrations greater than $\mathrm{C}_{\mathrm{T}}$. 


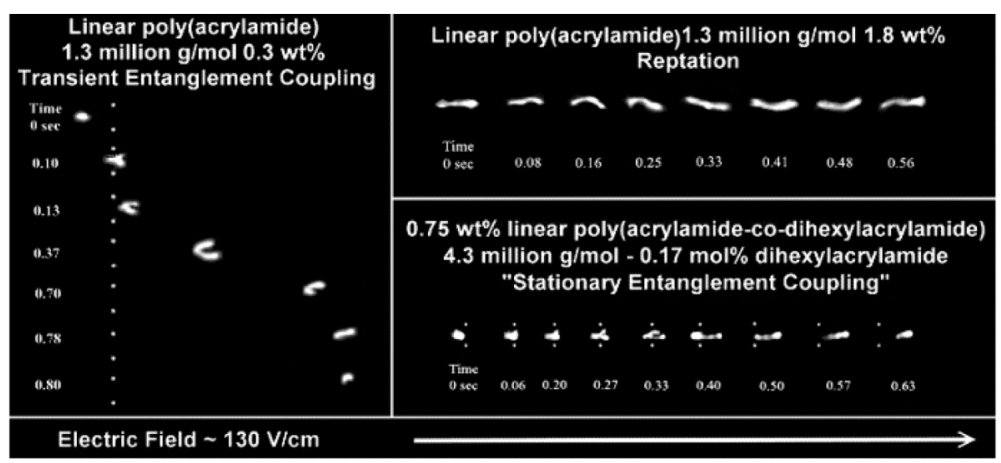

Figure 5.

Single-molecule epifluorescent videomicroscopy still-frame captures at indicated time points depicting the motion of DNA through $0.3 \mathrm{wt} \%$ LPA (left), $1.8 \mathrm{wt} \%$ LPA (top), and 0.75 wt \% LPA-co-DHA with 0.17 mol\% DHA (bottom). Three distinct DNA separation mechanisms can be seen: transient entanglement coupling (left), reptation (top), and the novel stationary entanglement coupling mechanism which resembles an "inchworm" motion (bottom). The applied electric field in each case is $\sim 130 \mathrm{~V} / \mathrm{cm}$, and the white dots have been superimposed over the video to act as reference points. 